\title{
Morbidity during the first year of life in small for gestational age infants
} Torstein Vik, Lars Vatten, Trond Markestad, Gunnar Ahlsten, Geir Jacobsen,
Leiv S Bakketeig mothers with a previous normal birthweight child (non-repeaters). ${ }^{816} 18$

SGA infants may also be classified as symmetric or asymmetric according to body proportions. $^{138}{ }^{19-21}$ Symmetric SGA neonates have normal body proportions; those that are asymmetric have a lower weight in relation to length. ${ }^{1319}{ }^{21}$ Symmetric SGA infants may be constitutional or the result of intrauterine environmental exposure starting in early pregnancy. Conversely, asymmetric SGA infants may be the result of placental insufficiency in late pregnancy. ${ }^{1-3} 1920$ Some investigators have found higher neonatal morbidity in asymmetric than in symmetric SGA infants, ${ }^{1-3} 222329$ while others have failed to demonstrate such differences. ${ }^{124}$

It is important to exclude preterm SGA and infants with congenital malformations from the analysis. Otherwise, SGA morbidity may be related to disorders associated with prematurity and congenital malformations, and not to the possible adverse effects of being born small for gestational age.

We have reported that newborn SGA infants with asymmetric body proportions were more often transferred to the neonatal intensive care unit than non-SGA infants. ${ }^{23}$ In this study we now present a further follow up of these infants, the aim being to compare morbidity during the first year of life between SGA and non-SGA infants born at term who had no congenital malformations.

vs;1Small for gestational age (SGA) infants have higher rates of perinatal morbidity and mortality than appropriate for gestational age infants. $^{1-3}$ Most follow up studies of SGA infants have focused on growth and mental and motor development. ${ }^{45}$ It has been reported that SGA infants are at risk for the development of cerebral palsy ${ }^{67}$ and minor neurological diseases including minimal brain dysfunction and speech and learning impairment. ${ }^{45}$ Associations between SGA and sudden infant death syndrome (SIDS) ${ }^{89}$ and early onset of insulin dependent diabetes mellitus have been reported. ${ }^{10}$ Low birthweight has also been associated with increased risk of cardiovascular disease in adult life. ${ }^{11}$ Few studies, however, have reported information on infant morbidity after the neonatal period. ${ }^{12-14}$

Some SGA infants are born to mothers who repeatedly give birth to low birthweight infants (repeaters), ${ }^{815-17}$ and epidemiological studies have indicated that these infants have a lower perinatal mortality than SGA infants born to

\section{Methods}

This study is part of a population based prospective multicentre study. Eligible for enrolment were para 1 and para 2 women of Caucasian origin in Trondheim and Bergen, Norway, and in Uppsala, Sweden, who had a singleton pregnancy, and were registered by one of the study centres before the 20th gestational week. Women were enrolled between January 1986 and March 1988. Further details have been reported before. ${ }^{25}$

SMALI FOR GESTATIONAL AGE (SGA) INFANTS Among a total of 6354 women referred to the study, 5722 were eligible to participate. Overall, 541 infants born at term (from 37 to 42 weeks of gestation) satisfied the criteria of being SGA, defined as a birthweight below the 10th percentile for gestational age, corrected for sex and parity. ${ }^{25}$ Twenty nine SGA infants were excluded due to congenital malformations $(n=22)$, stillbirth $(n=3)$, and death 
Table 1 Comparison between infants included in follow up from birth to 13 month examination and infants not included (Numbers are mean and standard deviation)

\begin{tabular}{|c|c|c|c|}
\hline & Included & Not included & $P$ value \\
\hline \multicolumn{4}{|l|}{ Non-SGA infants: } \\
\hline Number of infants & 359 & 102 & \\
\hline Birthweight (g) & $3773 \quad(468)$ & $3734 \quad(421)$ & 0.4 \\
\hline Length at birth $(\mathrm{cm})$ & $51.3(1.9)$ & $51.5(1.8)$ & 0.4 \\
\hline Head circumference at birth $(\mathrm{cm})$ & $35.5(1.2)$ & $35.3(1.6)$ & 0.2 \\
\hline Ponderal index & $2.79(0.23)$ & $2.74(0.23)$ & 0.03 \\
\hline \multicolumn{4}{|l|}{ SGA infants: } \\
\hline Number of infants & 284 & 82 & \\
\hline Birthweight (g) & 2888 & 2858 & 0.5 \\
\hline Length at birth $(\mathrm{cm})$ & $48.4(1.6)$ & (2.1) & 0.4 \\
\hline Head circumference at birth $(\mathrm{cm})$ & 33.9 (1.1) & 33.7 (1.5) & 0.4 \\
\hline Ponderal index & $2.54(0.20)$ & $2.48(0.26)$ & 0.06 \\
\hline
\end{tabular}

Table 2 SGA, non-SGA, and subgroups of SGA infants admitted to hospital during infancy (excluding first week of life) and during first week of life (Odds ratio (OR) and 95\% confidence interval (CI) are calculated for comparison with non-SGA infants)

\begin{tabular}{llll}
\hline & No & Yes & OR (95\% CI) \\
\hline Admitted during infancy & & & \\
SGA: & 232 & 52 & $1.7(1.1-2.6)$ \\
$\quad$ Repeaters & 99 & 14 & $1.1(0.6-2.0)$ \\
Non-repeaters & 119 & 37 & $2.4(1.4-3.8)$ \\
Symmetric & 144 & 38 & $2.0(1.2-3.3)$ \\
Asymmetric & 85 & 14 & $1.3(0.7-2.4)$ \\
Non-SGA & 317 & 42 & 1.0 \\
Admitted during first week of life & & & \\
SGA: & 326 & 32 & $1.7(1.0-2.9)$ \\
Repeaters & 126 & 16 & $1.3(1.1-4.2)$ \\
Non-repeaters & 177 & 14 & $1.1(0.5-2.2)$ \\
Symmetric & 209 & 11 & $3.2(1.7-6.0)$ \\
Asymmetric & 114 & 21 & 1.0 \\
Non-SGA & 427 & 25 & \\
\hline
\end{tabular}

during infancy ( $n=4$, sudden infant death syndrome). In all, 146 SGA infants were identified in retrospect, and were not included in this follow up. Infants who were identified as being SGA at birth were examined by a paediatrician allocated to this project. A total of 366 SGA infants were identified and registered for follow up during infancy. None of these infants had a diagnosis of congenital virus infection or toxoplasmosis.

An SGA infant whose mother had previously given birth to a low birthweight infant was classified as the child of a repeater. ${ }^{25}$ If the mother had not previously given birth to a low birthweight infant the child was classified as born to a non-repeater.

The ponderal index ((weight (grams)/ length $\left.(\mathrm{cm})^{3}\right) \times 100$ ) was used to divide the SGA infants into asymmetric or symmetric categories. ${ }^{26}$ SGA infants with a sex specific ponderal index below the 10th percentile (boys: 2.40, girls: 2.51) were defined as asymmetric, and those with a ponderal index at the 10th percentile or above were defined as symmetric SGA infants.

\section{CONTROL INFANTS}

Between week 17 and 20 of pregnancy, a $10 \%$ random sample was drawn from the population of 5722 women. Among a total of 561 women in this random sample, 532 delivered at term and $58(11 \%)$ of these infants were classified as SGA. Four of these were excluded because of congenital malformations or death, as stated above. The remaining 54 infants were included in the group of SGA infants. Of the remaining non-SGA infants, 15 were excluded due to congenital malformations $(n=12)$, perinatal $(n=1)$, or infant $(n=2$, sudden infant death syndrome) death, leaving a total of 459 infants to be followed up in the control group.

Data on early neonatal morbidity of the SGA and non-SGA infants have been reported before. ${ }^{23}$ To compare infant morbidity and neonatal morbidity, some data from the neonatal study are also presented in this paper.

FOLLOW UP

During their first year of life, all the children were examined by a public health nurse as part of the routine health service offered to all infants in Norway and Sweden. Data on health, growth, nutrition, development and behaviour were recorded at 6 weeks and at 3, 6, and 9 months of age. At each visit, the mother was asked if the infant had had any kind of disease or if the child had been admitted to hospital since the previous visit. The mother was asked to specify any disease, and if the child had been admitted to hospital she was asked to state the reason. After each visit the records were sent to the project organiser for data registration.

At 13 months, the infants were examined by a project paediatrician. Height, weight, head circumference and other anthropometric measurements were recorded and a general paediatric examination was performed. Data on growth and mental development will be published shortly. ${ }^{27}$ The mother was asked to complete a questionnaire on her child's health. She was asked if the infant had has respiratory or other infections, any other disorder of the respiratory system, disorders of the urinary and gastrointestinal tract, neurological, genital, or skin disorders, or any trauma during the first year of life. If the answer was positive, the mother was asked to give more information and to indicate how many times a physician had been consulted for each condition. Furthermore, the mother was asked if her child had been admitted to hospital during the preceding year, how many times, and for what reason(s).

If a mother had reported to the public health nurse that her infant had been admitted to hospital, or if she reported it in the 13 month questionnaire, the infant was classified as having been hospitalised during infancy. Admissions during the early neonatal period (the first week of life) were not recorded as infant morbidity.

Infants with data from at least two of the five examinations between 6 weeks and 13 months were included in the study. Complete follow up data were available for 284 SGA $(77.6 \%)$ and $359(78.2 \%)$ control infants. In all, $473(74 \%)$ had attended all visits to the public health nurse and the paediatric examination at 13 months, and another 138 infants $(21 \%)$ had attended all but one. In less than 5\%, two (24 infants) or three (8 infants) examination data were missing. There was no difference in missing data between SGA and non-SGA infants.

Of the 284 SGA infants who were included in the follow up, $113(40 \%)$ were born to repeaters and 156 to non-repeaters, and there was no information on previous outcome for 15 mothers. Among the SGA infants who 
Table 3 Bivariate analysis of admission to hospital of SGA and non-SGA infants during infancy stratified for $S G A$ risk factors (Odds ratio (OR) and $95 \%$ confidence interval are calculated for comparison of $S G A$ with non-SGA infants)

\begin{tabular}{|c|c|c|c|c|c|}
\hline \multirow[b]{3}{*}{ Risk factors } & \multicolumn{2}{|l|}{$S G A$} & \multicolumn{2}{|c|}{ Non-SGA } & \multirow[t]{3}{*}{ OR $(95 \% C I)$} \\
\hline & \multicolumn{4}{|c|}{ Admission to hospital } & \\
\hline & No & Yes & No & Yes & \\
\hline \multicolumn{6}{|l|}{ Smoking: } \\
\hline Yes & 129 & 40 & 102 & 16 & $2.0(1.0-3.7)$ \\
\hline No & 102 & 12 & 214 & 26 & $1.0(0.5-2.1)$ \\
\hline \multicolumn{6}{|c|}{ Prepregnancy weight: } \\
\hline$<50 \mathrm{~kg}$ & 36 & 8 & 13 & 4 & $0.7(0.2-2.0)$ \\
\hline$\geqslant 50 \mathrm{~kg}$ & 196 & 44 & 304 & 37 & $1.8(1.1-3.0)$ \\
\hline \multicolumn{6}{|c|}{ Prepregnancy body mass } \\
\hline \multicolumn{6}{|c|}{ index $\left(\mathrm{kg} / \mathrm{m}^{2}\right)$ : } \\
\hline$<21$ & 134 & 34 & 125 & 20 & $1.6(0.9-2.9)$ \\
\hline$\geqslant 21$ & 98 & 18 & 191 & 20 & $1.8(0.9-3.5)$ \\
\hline \multicolumn{6}{|c|}{ Mother's education: } \\
\hline$\leqslant 9$ years & 82 & 23 & 135 & 17 & $2.2(1.1-4.4)$ \\
\hline$>9$ years & 62 & 17 & 181 & 25 & $2.0(1.3-3.9)$ \\
\hline
\end{tabular}

Table 4 SGA and non-SGA infants admitted to hospital during infancy (excluding first week of life), and in subgroups of SGA infants stratified for smoking (Odds ratio (OR) and $95 \%$ confidence interval (CI are calculated for comparison with non-SGA infants)

\begin{tabular}{lrrr} 
& \multicolumn{2}{c}{ Admitted to hospital } & \\
\cline { 2 - 3 } & No & Yes & OR $(95 \% C I)$ \\
\hline Non-smokers: & & & \\
SGA: & 102 & 12 & $1.0(0.5-2.1)$ \\
Repeaters & 43 & 4 & $0.8(0.3-2.3)$ \\
Non-repeaters & 57 & 8 & $1.1(0.5-2.6)$ \\
Symmetric & 61 & 8 & $1.1(0.5-2.6)$ \\
Asymmetric & 40 & 4 & $0.8(0.3-2.6)$ \\
Non-SGA & 214 & 26 & 1.0 \\
Smokers: & 129 & 40 & \\
SGA: & 56 & 10 & $2.0(1.0-3.7)$ \\
Repeaters & 62 & 29 & $1.4(0.6-3.2)$ \\
Non-repeaters & 83 & 30 & $3.1(1.6-6.1)$ \\
Symmetric & 45 & 10 & $2.3(1.2-4.5)$ \\
Asymmetric & 102 & 16 & $1.4(0.7-3.1)$ \\
Non-SGA & & & 1.0 \\
\hline
\end{tabular}

completed follow up, 99 (35\%) were classified as asymmetric and 182 infants as having symmetric body proportions, while the ponderal index could not be calculated for three SGA infants.

In table 1 we have compared the perinatal data of infants with and without complete follow up information. There was a small difference in ponderal index between the groups, but there were no differences in the proportions of repeaters/non-repeaters or in symmetric/asymmetric SGA infants (data not shown).

Gestational age was estimated from the first day of the last menstrual period if this was accurately recalled by the mother within more than three days at the first visit. Ultrasound estimates were used for dating in about $17 \%$ of the cases, either due to a discrepancy between the date of the last menstrual period and ultrasound based gestational age of more than 14

Table 5 Causes of admission to hospital during first year of life (1 week to 13 months) in $S G A$ and non-SGA infants (Odds ratio (OR) and $95 \%$ confidence interval (CI) are calculated for comparison of $S G A$ with non-SGA infants)

\begin{tabular}{lrrl}
\hline & $S G A$ & Non-SGA & $O R(95 \%$ CI) \\
\hline Infections: & 32 & 21 & $2.0(1.1-3.8)$ \\
Respiratory tract infections & 21 & 12 & $2.2(1.1-4.4)$ \\
Other infections & 11 & 9 & $1.6(0.6-4.2)$ \\
Surgery & 8 & 4 & $2.6(0.7-10.3)$ \\
Poor weight gain/feeding difficulties & 2 & 3 & $0.8(0.1-6.2)$ \\
Other diagnoses & 10 & 14 & $0.9(0.4-2.2)$ \\
Total & 52 & 42 & $1.7(1.1-2.6)$ \\
\hline
\end{tabular}

days, or if the last menstrual period could not be recalled accurately. ${ }^{25}$

Mothers who reported smoking daily at the time of conception were classified as smokers. Other potential risk factors for SGA which were considered included a prepregnancy weight below $50 \mathrm{~kg}$, a body mass index below the median value of $21 \mathrm{~kg} / \mathrm{m}^{2}$, and less than 10 years of education.

The odds ratio was used to estimate the relative risk of morbidity among SGA compared with non-SGA infants. Precision of the odds ratio was estimated by $95 \%$ confidence intervals, using Mantel-Haenszel $\chi^{2}$ statistics.

\section{Results}

A total of 94 infants were admitted to hospital during the first year of life, excluding the first week, and SGA infants were more often admitted than non-SGA infants (OR: $1.7 ; 95 \% \mathrm{CI}$ : 1.1-2.6) (table 2). Nine mothers, all in the SGA group, reported that their child had been admitted to hospital twice. After excluding readmissions of infants who had been transferred to the intensive care unit during the neonatal period, the increased risk of being admitted to hospital persisted in SGA infants (OR: 1.6, 95\% CI: 1.0-2.5).

The higher admission rate of SGA infants was mainly found among SGA infants of mothers who were non-repeaters and in SGA infants with symmetric body proportions at birth (table 2).

For comparison, admissions during the first week of life are shown in table 2. Newborn SGA infants had a higher risk of being admitted to hospital than non-SGA infants, but the higher admission rate in the early neonatal period was mainly caused by infants of repeaters and in infants with asymmetric body proportions.

Table 3 shows the odds ratios of being admitted to hospital in SGA and non-SGA infants stratified by maternal risk factors. Among infants of mothers who smoked, there was a twofold increased risk of being admitted to hospital for SGA compared with non-SGA infants. Among infants of non-smokers, there was no such difference between SGA and nonSGA infants.

Among infants of mothers with a prepregnancy weight below $50 \mathrm{~kg}$, being SGA was not associated with an increased risk of being admitted to hospital (table 3 ). The relative risk for SGA infants was, however, increased if the mother had a prepregnancy weight above 50 kg.

The higher admission rate among SGA infants of smokers was caused by infants of non-repeaters and by infants with symmetric body proportions (table 4). Among nonsmokers, no evidence of increased risk was found within subgroups of SGA (table 4).

Table 5 shows that infections more often led to admission to hospital among SGA than non-SGA infants (OR: 2.0, 95\% CI: 1.1-3.8). This was mainly due to a higher incidence of respiratory tract infections (OR: $2.2,95 \% \mathrm{CI}$ : 1.1-4.4) 
Upper respiratory tract infections (common cold, pharyngitis, and otitis media) were reported during the first year of life by $91.5 \%$ of the SGA and 91.4 of the non-SGA mothers. However, exanthema subitum and varicella were reported significantly more often in the non-SGA (70 infants; $20 \%$ ) than in the SGA (35 infants; $12 \%$ ) group (OR: 1.7, 95\% CI: 1.1- 2.7). In both groups $55 \%$ of the mothers reported that they had consulted a physician during the preceding year (mean: 1.3 contacts per infant and year in both groups).

At the clinical examination at 13 months, the paediatricians reported pathological findings in more than 10 infants only for the examination of the ears ( 25 infants), lungs (12 infants), and skin (55 infants). For SGA compared with non-SGA infants the odds ratios for pathological findings of the ears and lungs (combined) were 1.7 (95\% CI: 0.8-3.4) and for pathology of the skin 0.7 (95\% CI: 0.4-1.2).

\section{Discussion}

We found that SGA infants had an increased risk of being admitted to hospital during infancy compared with non-SGA infants. Our study was restricted to singletons born at term with no congenital malformations and therefore the results were not caused by diseases related to prematurity, congenital malformations, or multiple births. Nor was the higher admission rate caused by a higher morbidity of infants who had been admitted to the neonatal intensive care unit during the newborn period.

The 10th percentile definition of SGA is crude, and many constitutionally small, but healthy, babies will be classified as SGA. ${ }^{28}$ None the less we found that SGA infants had a $70 \%$ increased risk of being admitted to hospital compared with non-SGA infants. A more narrow definition of SGA would most probably have resulted in a higher risk.

The outcome measure, admission to hospital, is likely to have been correctly recalled by the mother. The infants were admitted by many different general practitioners and it is unlikely that they were influenced by the child's SGA status when deciding on admission.

Most follow up studies of term SGA infants have not reported infant morbidity, except for persistent deficits such as cerebral palsy and neurological 'soft signs'. ${ }^{3-5}$ One study found no differences in postneonatal morbidity between SGA and non-SGA infants, ${ }^{13}$ whereas Villar $e t$ al reported an increased incidence of diarrhoea in symmetric SGA infants compared with nonSGA infants during the first two months of life. ${ }^{12}$ In a population based study from Brazil, hospital admissions due to diarrhoea and pneumonia occurred more often during the first two years in children who were growth retarded at birth than in children with appropriate birthweights. ${ }^{14}$

Among subgroups of SGA infants, we found an increased risk of hospital admission for infants of non-repeater mothers. The rationale for comparing repeaters and non-repeaters was the hypothesis that an "unexpected" adverse event or exposure during pregnancy may have caused the birth of an SGA infant to a non-repeater. ${ }^{6}$ SGA infants of repeaters may more often be constitutionally small, ${ }^{816} 18$ but may also be the result of an unhealthy lifestyle in consecutive pregnancies. ${ }^{16}$ Our results are consistent with those of studies which reported increased perinatal morbidity and mortality among SGA infants of non-repeaters. ${ }^{81618}$

By using the 10th percentile of the ponderal index as a cutoff between symmetric and asymmetric SGA, we found that symmetric SGA infants had a higher risk of being admitted to hospital. Symmetric SGA infants may either be constitutional, or the result of retarded intrauterine growth which may be caused by early environmental exposure. ${ }^{351920}$ Thus our results suggest that infant morbidity might be more affected in SGA infants who have been exposed to environmental factors in early pregnancy.

We stratified the infants according to risk factors for SGA and found no difference in the risk of being admitted to hospital between SGA and non-SGA infants of mothers with a low prepregnancy weight. However, the total number of non-SGA infants in this group was low $(n=17)$.

We analysed infants of smokers and nonsmokers separately and found that SGA infants of women who smoked at the beginning of pregnancy had an increased risk of being admitted to hospital. Among non-smokers there was no difference between SGA and non-SGA infants. Thus the increased risk of SGA infants of being admitted to hospital in this study was solely observed among SGA infants of smokers.

Smoking may lead to symmetric growth retardation, ${ }^{29}$ and for SGA infants of smokers we found a threefold increased risk of being admitted to hospital if the mothers were non-repeaters, compared with non-SGA infants. There was no increased risk for SGA infants of non-repeaters who did not smoke.

We found that infections more often led to hospitalisation of SGA than non-SGA infants. This was caused by a higher admission rate for respiratory tract infections. However, we found no increase in morbidity as a result of minor illnesses among SGA infants, and the reported prevalence of respiratory tract infections not leading to hospitalisation did not differ between the groups, suggesting that infections may lead to more severe disease in SGA than in non-SGA infants. This may be mediated through maternal smoking which has been shown to increase the risk of respiratory complications in infancy. ${ }^{30-33}$

Compared with our results from the early neonatal period, there was a similar increased risk of being admitted to hospital among SGA infants as a whole, ${ }^{23}$ but there were different patterns of hospitalisation among subgroups of SGA infants. Asymmetric SGA infants and infants of repeaters had an increased risk in the early neonatal period; later in infancy the increased risk was found among symmetric infants and among infants of non-repeaters.

The different patterns may be related to different reasons for admission. In the early 
neonatal period acute complications-for example, hypoglycaemia, hyperviscosity-were the principal causes of admission, ${ }^{23}$ and this is consistent with other reports. ${ }^{1-35}$ These causes are related to poor placental function late in pregnancy and to asymmetric body proportions at birth. After the neonatal period the asymmetric SGA infant may have recovered. The higher risk of being admitted to hospital in symmetric infants of non-repeating mothers, lasting for the remaining part of infancy, may be caused by earlier exposure with permanent effects on the infant's health.

In conclusion, we have found a higher prevalence of being admitted to hospital among SGA infants compared with non-SGA infants during infancy, which was restricted to symmetric SGA infants of non-repeating, mothers who smoked. The results may suggest that an exposure which influences living conditions in utero may have acted from early pregnancy and resulted in long term consequences for infant morbidity, particularly if the mother smokes cigarettes.

This study was supported by the National Institute of Child Health and Human Development, NIH (NICHD Contract No 1-HD-4-2803).

1 Lockwood CJ, Weiner S. Assessment of fetal growth. Clin Perinatol 1986;13:3-35.

2 Pearce JM, Campell S. Intrauterine growth retardation. Birth Defects 1985;21:109-30.

$3 \mathrm{Lin} \mathrm{CC.} \mathrm{Intrauterine} \mathrm{growth} \mathrm{retardation.} \mathrm{Obstet} \mathrm{Gynecol}$ Annu 1985;14:127-221.

4 Allen MC. Developmental outcome and follow-up of the small for gestational age infant. Semin Perinatol 1984; 8:123-56

5 Villar J, Belizan JM, Smeriglio V. Postnatal experiences of intrauterine growth-retarded infants. In: Senterre J ed. Intrauterine growth retardation. Raven Press. New York: 1989: 261-77.

6 Uvebrant P, Hagberg G. Intrauterine growth in children with cerebral palsy. Acta Paediatr 1992; 81:407-12.

7 Blair E, Stanley F. Intrauterine growth and spastic cerebral palsy. Am $\mathcal{F}$ Obstet Gynecol 1990; 162:229-37.

8 Hoffman HJ, Bakketeig LS. Heterogeneity of intrauterine growth retardation and recurrence risks. Semin Perinatol growth retardatic

9 Irwin KI, Mannino S, Daling J. Sudden infant death syndrome in Washington State: why are Native American infants at greater risk than white infants? $\mathcal{f}$ Pediatr 1992;121:242-7.

10 Khan N, Couper JJ. Low-birth-weight infants show earlier onset of IDDM. Diabetes Care 1994;17:653-6.

11 Barker DJP, Osmond C, Goldin J, Kuh D, Wadsworth MEJ. Growth in utero, blood pressure in childhood and early life and mortality from cardiovascular disease. $B M \mathcal{F} 1989$; 298:564-7.

12 Villar J, Smeriglio V, Martorelli R, Brown CH, Klein RE. Heterogeneous growth and mental development of intrauterine growth retarded infants during the first 3 years of terine growth retarded infants
life. Pediatrics 1984;74:783-91.
13 Downes B, Downes R, Foord F, Weaver L. Outcome of low birth weight infants in a West African village. $\mathcal{F}$ Trop Pediatr 1991;37:106-10.

14 Barros FC, Huttly SR, Victora CG, Kirkwood BR, Vaughan JP. Comparison of the causes and consequences of prematurity and intrauterine growth retardation: a longitudinal study in southern Brazil. Pediatrics 1992;90:238-44.

15 Ounsted M. Maternal constraint of foetal growth in man. Devel Med Child Neurol 1965;7:479-90.

16 Bakketeig LS, Bjerkedal T, Hoffman HJ. Small-forgestational age births in successive pregnancy outcomes: gestational age births in successive pregnancy outcomes: results from a longitudinal study

17 Goldenberg RL, Hoffman HJ, Cliver SP, Cutter GR, Nelson KG Copper RL. The influence of previous low birth weight on birth weight, gestational age, and anthropometric measurements in the current pregnancy. Obstet Gynecol 1992;79:276-80.

18 Read AW, Stanley FJ. A comparison of reccurent and isolated small-for-gestational-age term births. Paediatr Perinat Epidemiol 1991;5:138-56.

19 Villar J, Belizan JM. The timing factor in the pathophysiology of intrauterine growth retardation syndrome. Obstet ogy of intrauterine growth retard

20 Rosso $P$, Winick $M$. Intrauterine growth retardation. A new systemic approach based on the clinical and biochemical characteristics of this condition. F Perinat Med 1974;2:14760.

21 Walther FJ, Ramaekers LHJ. The ponderal index as a measure of the nutritional status at birth and its relation to some aspects of neonatal morbidity. $\mathcal{f}$ Perinat Med 1982;10:42-7.

22 Caulfield LE, Haas JD, Belizan JM, Rasmussen KM, Edmonston B. Differences in early postnatal risk by pattern of fetal growth in Argentina. Paediatr Perinat Epidemiol 1991;5:263-75.

23 Vik T, Markestad T, Ahlsten G, Gebre-Medhin M, Jacobsen G, Hoffman HJ, et al. Body proportions and early neonatal morbidity in small for gestational age infants of successive morthidity in small for gestational age infants of success

24 Balcasar H, Haas J Classification schemes of small-forgestational age and type of intrauterine growth retardation and its implication to early neonatal mortality. Early Hum Dev 1990;24:219-30.

25 Bakketeig LS, Jacobsen G, Hoffman HJ, Lindmark G, Bergsjø $\mathrm{P}$, Molne $\mathrm{K}$, et al. Pre-pregnancy risk factors of smallfor-gestational age births among parous women in Scandinavia. Acta Obstet Gynecol Scand 1993;72:273-9.

26 Rohrer R. Der Index der Körperfülle als Mass des Ernährungszustandes. Münch Med Wochenschr 1921; 68:580-3.

27 Markestad T, Vik T, Ahlsten G, Gebre-Medhin M, Skjærven $\mathrm{R}$, Jacobsen $\mathrm{G}$, et al. Small for gestational age (SGA) infants born at term: Growth and development during the first year of life. Acta Obstet Gynecol Scand (Suppl) (in press).

28 Chamberlain G. Small for gestational age. BMF 1991; 302:1592-6.

29 Miller H, Hassanein K, Hensleiger P. Fetal growth retardation in relation to maternal smoking and weight gain in pregnancy. Am 7 Obstet Gynecol 1976;125:55-60.

30 Graham NM. The epidemiology of acute respiratory infections in children and adults: a global perspective. Epidemiol tions in children and

31 McConnochie KM, Roghmann KJ. Parental smoking, presence of older siblings, and family history of asthma increase risk of bronchiolitis. Am $f$ Dis Child 1986; 140:806-12.

32 Taylor B. Prevention of pediatric pulmonary problems: the importance of maternal smoking. Lung 1990;168(Suppl): 327-32.

33 American Heart Association, Council on Cardiovascular Disease in the Young. Active and passive tobacco exposure: a serious pediatric health problem. A statement from the Committee on Atherosclerosis and Hypertension in Children. Circulation 1994;90:2581-90. 